Communications in Physics, Vol. 31, No. 3 (2021), pp. 279-287

DOI:10.15625/0868-3166/15668

\title{
AGGREGATION OF ZOOSPORES ON SHARKLET MICROTOPOGRAPHIC SURFACES
}

\author{
NHUNG T. T. NGUYEN ${ }^{1}$ AND TRINH XUAN HOANG ${ }^{1,2, \dagger}$ \\ ${ }^{1}$ Institute of Physics, Vietnam Academy of Science and Technology, \\ 10 Dao Tan, Ba Dinh, Hanoi, Vietnam \\ ${ }^{2}$ Physics Department, Graduate University of Science and Technology, \\ Vietnam Academy of Science and Technology, 18 Hoang Quoc Viet, Cau Giay, Hanoi, Vietnam \\ E-mail: †hoang@iop.vast.vn
}

Received 9 November 2020

Accepted for publication 5 April 2021

Published 18 April 2021

\begin{abstract}
Surfaces with engineered microtopographies are potential candidate against biofouling to replace the use of biocides in the marine environment. Understanding the antifouling mechanism of microtopographic surfaces against marine microorganisms, however, has been limited. In this work, we theoretically studied the aggregation of Ulva linza zoospores on the Sharklet topographic surfaces by employing the extended Surface Energetic Attachment (SEA) model proposed in a previous work. The energy parameters of the model were obtained by matching theoretical results with experimental data for one type of Sharklet surface. Monte Carlo simulations were then carried out for a series of Sharklet surfaces with various numbers of distinct features. In agreement with prior experimental results, our simulations indicate that engineered topographies promote smaller aggregates than those on a smooth surface. Furthermore, we show that the maximum effect of the Sharklet topography on the aggregate size of $U$. linza can be obtained with just 3 distinct features.
\end{abstract}

Keywords: biofouling; aggregation; extended SEA model; Monte Carlo simulation.

Classification numbers: 87.15.M-; 87.64.K-.

\section{INTRODUCTION}

The attachment of micro- and macro-organisms living in aqueous environments to submerged surfaces, known as biofouling, is a major problem in maritime transport [1-4]. Biofouling on a ship's hull can increase the hydrodynamic drag by $60 \%$ [4] leading to a loss of speed, a decrease in maneuverability, increased fuel consumption, thereby increased emissions of greenhouse (C)2021 Vietnam Academy of Science and Technology 
gases and increased cost of maintenance. The wide use of biocides, such as tributyltin (TBT), in antifouling paints on ships' hulls for many years had a severe effect on the environment $[5,6]$ (TBT was banned internationally in 2003). Among the non-toxic antifouling strategies being developed, the coating method based on engineered microtopographic surfaces is promising to replace the use of chemicals in the marine environment [1]. This method increases the roughness of a surface by introducing repeated patterns of micrometer-sized protruded features to obtain an antifouling effect. Various patterns with features like pillars, squares, triangles, ridges etc. have been considered in experiments. An interesting pattern which shows an enhanced antifouling property is the bioinspired Sharklet pattern, for that it mimics the topography of shark skin [3]. It has been shown that a Sharklet topographic surface can reduce the attachment of zoospores by $86 \%$ compared to a smooth surface [7].

A number of theories have aimed to predict the attachment behaviors of marine microorganisms on topographic surfaces. The attachment point theory (APT) [8-10] is based on the observation that the organisms preferentially bind to locations where they can maximize the number of contact points with a surface. The engineered roughness index (ERI) model [11] relates the attachment densities to a surface roughness index which was derived based on the theories of wetting $[12,13]$. The surface energetic attachment (SEA) model [14] assigns attachment energies to the binding of an organism to different site types on a surface and determines the attachment probabilities from the Boltzmann's statistics. The SEA model can predict attachment probabilities on various topographies, but is limited only to low density foulings. A recent study [15] has extended the SEA model to incorporate inter-organism interactions and employed statistical mechanics methods to calculate the equilibrium properties of systems of multiple spores on a topographic surface. The extended SEA model is applicable to high density foulings and can be used to study the aggregation of microorganisms on topographic surfaces. Note that a common tendency of microorganisms to live as social communities, thus they typically form aggregates or biofilms on surfaces to which they attach $[16,17]$.

In the present study, we investigated the aggregation of microorganisms on topographic surfaces using the extended SEA model. Specifically, we carried out Monte Carlo simulations within this model for the fouling of Ulva linza zoospores on a series of Sharklet topographic surfaces with different number of distinct features as well as on a smooth surface. The aim of the study is to see how surface topography affects the formation of aggregates and whether any quantitative prediction can be obtained from the simulations.

\section{MODELS AND METHODS}

Consider Sharklet surfaces of multiple unit cells. A Sharklet unit cell with four distinct features is shown in Fig. 1(a), in which the features are of rectangular shapes and are placed parallel to each other. In the present study, the feature width and feature spacing are fixed and equal to $2 \mu \mathrm{m}$, thus the features are different only by their lengths. The feature height is not relevant in our consideration. Let's denote $n$ the number of distinct features. The surfaces are discretized on a two-dimensional square lattice of $1 \mu \mathrm{m}$ lattice spacing (Fig. 1(b)). The lattice sites are divided into three types depending on whether it is on top of a feature $(t=1)$, between the long edges of two different features $(t=2)$ or between the corners of different features $(t=3)$. Let $g_{t}$ be the number of lattice sites of type $t$ in the unit cell. The fouling microorganisms, the spores in our case, are modelled as spheres of diameter $d=5 \mu \mathrm{m}$ (shown as circles in Fig. 1(c)). 


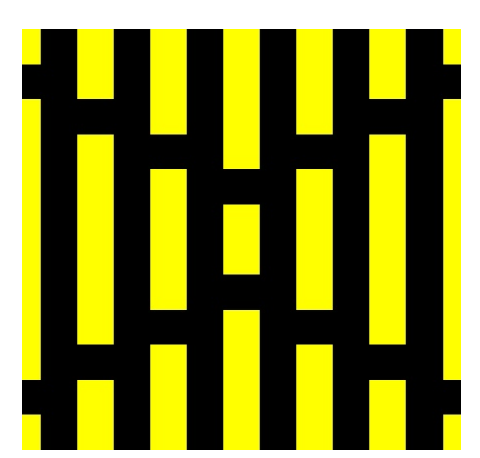

(a)

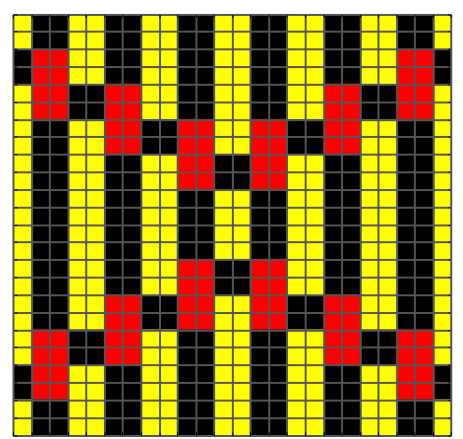

(b)

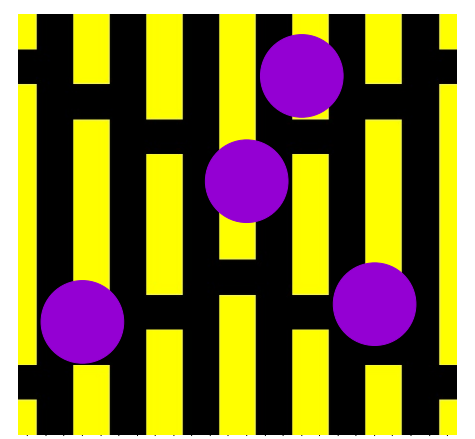

(c)

Fig. 1. (a) A Sharklet unit cell with $n=4$ distinct features. The feature tops are shown with yellow color whereas the depressed spaces between features are shown in black color. The feature width and feature spacing are equal to $2 \mu \mathrm{m}$. (b) Disretization of the Sharklet unit cell on a square lattice of $1 \mu \mathrm{m}$ lattice constant. The lattice sites are of three different types denoted as $t=1$ (yellow), $t=2$ (black) and $t=3$ (red). (c) A configuration of 4 spores shown as filled circles of $5 \mu \mathrm{m}$ diameter (purple) attached to the unit cell.

In the SEA model [14], the spores do not interact with each other. Each spore is assigned an attachment energy $E_{t}$ which depends on the type $t$ of the lattice site to which it attaches. Let $N_{t}$ be the number of spores attached to the type $t$ 's sites, and $N=\sum_{t} N_{t}$ be the total number of spores. Because the spores do not interact, the attachment probability $p_{t}$ for a given $t$ is obtained as in a single spore system, i.e.

$$
p_{t}=\frac{\left\langle N_{t}\right\rangle}{N}=\frac{g_{t} e^{-E_{t} / k_{B} T}}{Q_{1}} \quad \text { with } \quad Q_{1}=\sum_{t=1}^{3} g_{t} e^{-E_{t} / k_{B} T},
$$

where $\left\langle N_{t}\right\rangle$ is the average number of spores on the $t$ sites, $Q_{1}$ is the 1-spore partition function, $k_{B}$ is the Boltzmann's constant and $T$ is the absolute temperature. From Eq. (1), it follows that for any pair of site types $t$ and $u$

$$
\log \left(\frac{p_{t}}{p_{u}}\right)=\log \left(\frac{g_{t}}{g_{u}}\right)-\frac{E_{t}-E_{u}}{k_{B} T}
$$

Eq. (2) indicates that the attachment probabilities depend only on the energy difference between different site types. Eq. (2) can be used to determine the energy parameters $E_{t}$ from experimental attachment probabilities, as well as to predict these probabilities once the energy parameters have been determined.

The extended SEA model [15] added inter-organism interactions to the SEA model. First, the spores have an excluded volume interaction meaning that the center-to-center distance between any two spores cannot be smaller than $d$. Second, the spores can also have energetic contacts with each other. Two spores are said to make a contact if their center-to-center distance is smaller than a cut-off distance taken to be $6 \mu \mathrm{m}$, and an energy $E_{\text {int }}$ is assigned to each contact. For a system of $N$ spores on a surface, the total energy in a configuration $\Gamma$ is given by

$$
E(\Gamma)=\sum_{t} N_{t} E_{t}+N_{\mathrm{int}} E_{\mathrm{int}},
$$


where $N_{t}$ is the number of spores residing on the type $t$ 's lattice sites and $N_{\text {int }}$ is the number of spore-spore contacts. The probability of observing the configuration $\Gamma$ is given by that of the canonical ensemble

$$
p(\Gamma)=\frac{e^{-E(\Gamma) / k_{B} T}}{Q_{N}} \quad \text { with } \quad Q_{N}=\sum_{\Gamma} e^{-E(\Gamma) / k_{B} T},
$$

where the summation for the $N$-spore partition function $Q_{N}$ is taken over all possible spore configurations on the lattice. The average of a quantity $A$ in the ensemble is given by

$$
\langle A\rangle=\sum_{\Gamma} A(\Gamma) p(\Gamma) .
$$

Monte Carlo (MC) simulations were carried out to calculate the averages given by Eq. (5). For a given spore system, a MC simulation started from a random configuration of spores on the lattice. In each MC step, a spore is attempted to move to a randomly chosen new position on the lattice. The move was rejected if the new position caused a steric class of the spore, otherwise it was accepted with a probability $P=\min \left\{1, e^{-\Delta E / k_{B} T}\right\}$ by the Metropolis algorithm [18], where $\Delta E=E^{\prime}-E$ is the energy difference between the new and the old configurations. Periodic boundary conditions were applied to both directions of the square lattice.

\section{RESULTS AND DISCUSSION}

To study the aggregation of $U$. linza zoospores on the Sharklet surfaces, we first inferred the energy parameters $E_{t}$ in the SEA model from experimental data [19] by using Eq. (2). The experiment carried out by Long et al. [19] for $U$. linza has reported that on the Sharklet $n=4$ surface, $96 \%$ of the spores attached on the depressed regions ( $t=2$ and $t=3$ sites), of which $56 \%$ attached on the intersection regions $(t=3$ sites). These statistics were obtained for individual spores, i.e. those do not form aggregates. From these statistics, one finds the attachment probabilities $p_{1}=0.04, p_{2}=0.4$, and $p_{3}=0.56$ for the three site types $t=1,2,3$. By using Eq. (2), one obtains $E_{1}=0, E_{2}=-2.303 k_{B} T$ and $E_{3}=-3.555 k_{B} T$ ( $E_{1}$ was fixed to zero because only the energy difference matters). The obtained energy parameters $E_{t}$ were then used for the simulations in the extended SEA model. The spore-spore interaction energy $E_{\text {int }}$ was determined by matching the simulation results on the aggregate size to the experimental result in Ref. [19] for the Sharklet $n=4$ surface.

Systems of $N=100$ spores were simulated on the Sharklet surfaces with the number of distinct features $n=1,2, \ldots 7$. For comparison, we ran also simulations for a smooth surface which is denoted by $n=0$. The size of the surfaces with $n=0,1,2,3,4$ was chosen to be equal to $240 \mu \mathrm{m} \times 240 \mu \mathrm{m}$, corresponding to an integer number of unit cells in each dimension (10 by 10 unit cells for $n=4$ ). The overall spore density is 1,736 spores $\mathrm{mm}^{-2}$, which is comparable to the high fouling density in experiments. For $n=5,6,7$ the surface dimensions and also the number of spores were slightly modified such that the number of unit cells remains integer and the surface area and the overall spore density are most close to those of lower $n$ 's. For example, with $n=5$ the dimensions are $224 \mu \mathrm{m} \times 252 \mu \mathrm{m}$ (7 by 9 unit cells) and $N=98$. In each simulation, a system was first equilibrated for $10^{6} \mathrm{MC}$ sweeps before being sampled under equilibrium conditions over the next $10^{6} \mathrm{MC}$ sweeps. The averages were typically taken over 50 independent MC simulations. 
We first examined the dependence of the average attachment numbers $\left\langle N_{t}\right\rangle$ on the energy parameter $E_{\text {int }}$ for the fouling on the Sharklet $n=4$ surface. Fig. 2 shows that for all three site types, the fractions $\left\langle N_{t}\right\rangle / N$ are almost constant for $E_{\text {int }}>-2 k_{B} T$ with highest attachment number belong to the $t=3$ sites and the lowest to the $t=1$ sites. The attachment numbers however change drastically when $E_{\text {int }}$ becomes lower than $-2.8 k_{B} T$ and at some point the attachment number for the $t=3$ sites becomes lower than that for the $t=1$ sites. These changes are associated with the formation of large aggregates on the surface and are akin to a first-order phase transition in 2D [20]. Figure 3 shows that the aggregate size distribution strongly depends on $E_{\text {int }}$.

The value of $E_{\text {int }}$ was selected such that it gives the best agreement between simulations and experiments on the characteristics of the aggregates. A quick comparison of the aggregate size distributions in Fig. 3 to those reported by Decker et al. [21] for $U$. linza on the Sharklet $n=4$ surface (Fig. 4 in Ref. [21]) gave us a roughly estimated $E_{\text {int }}=-2.8 k_{B} T$. We then used this value of $E_{\text {int }}$ for the analyses of fouling on other surfaces. Note that this value of $E_{\text {int }}$ is close to the attachment energies $E_{2}$ and $E_{3}$ for the site types $t=2$ and $t=3$.

Figure 4 shows examples of equilibrium spore configurations obtained on the smooth and several Sharklet surfaces with $E_{\text {int }}=-2.8 k_{B} T$. One can see that the surface topography strongly influences the aggregation behavior. The spores tend to form a large aggregate on the smooth surface and much smaller aggregates on the Sharklet's $n=2$ and $n=4$ surfaces. The $n=1$ case seems to correspond to a transition point in this trend.

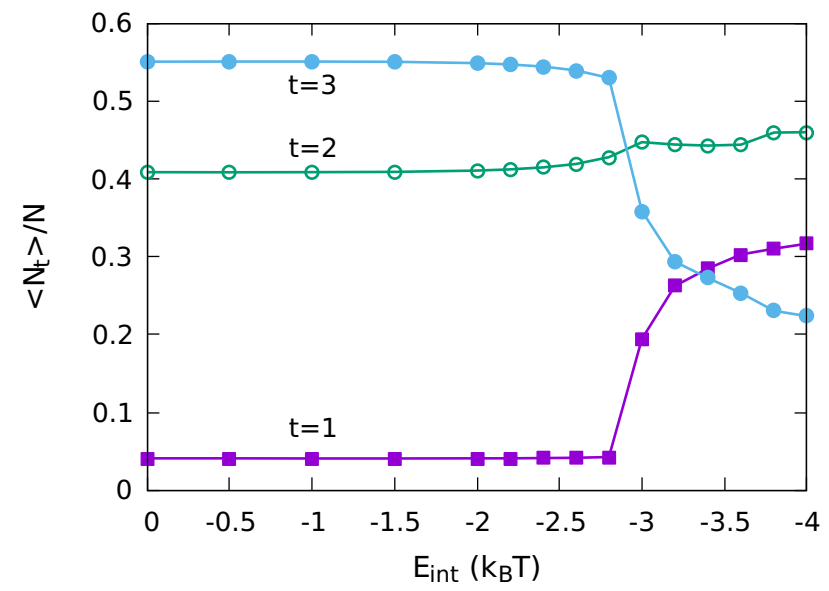

Fig. 2. Dependence of the fractional attachment numbers $\left\langle N_{t}\right\rangle / N$ on the inter-organism interaction energy parameter $E_{\text {int }}$ for a system of $N=100$ spores on the Sharklet $n=4$ surface.
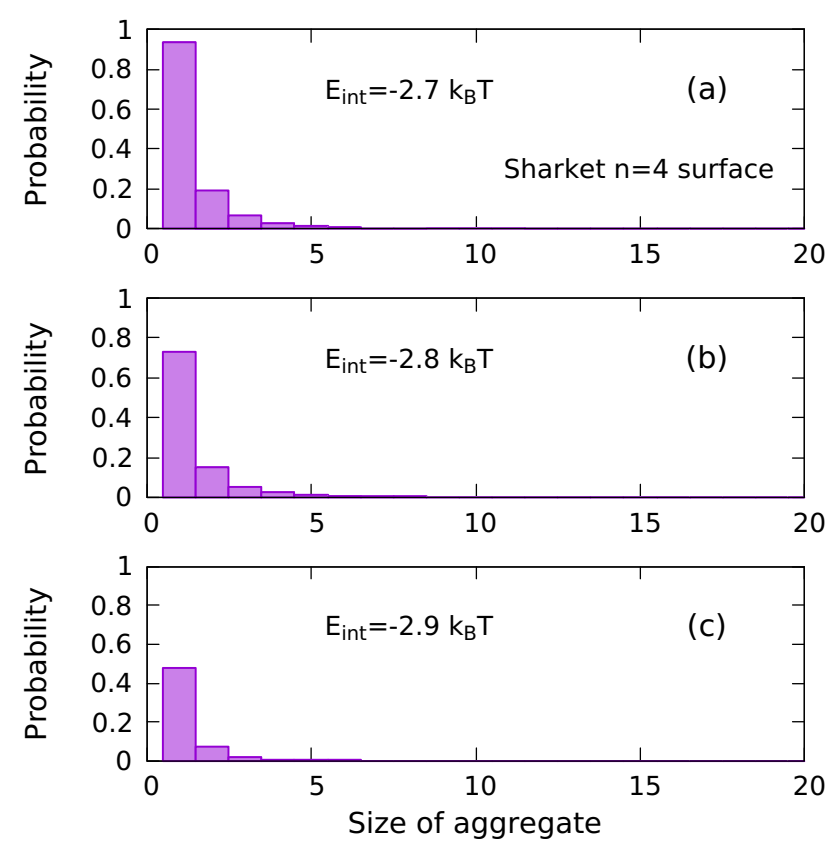

Fig. 3. Aggregate size distributions for systems of $N=100$ spores on the Sharklet $n=4$ surface obtained by the simulations with $E_{\text {int }}=-2.7 k_{B} T$ (a), $E_{\text {int }}=-2.8 k_{B} T$ (b), and $E_{\text {int }}=-2.9 k_{B} T$ (c). 


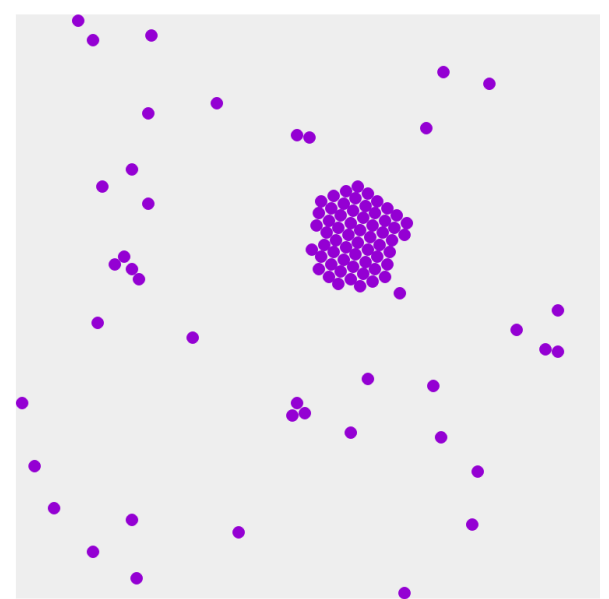

(a)

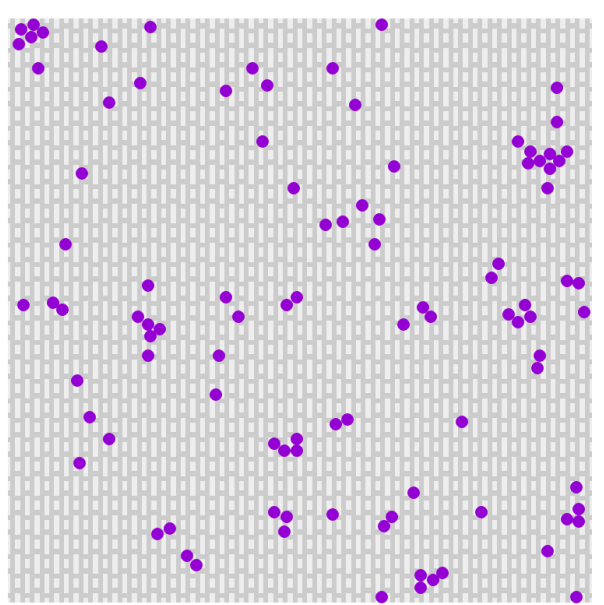

(c)

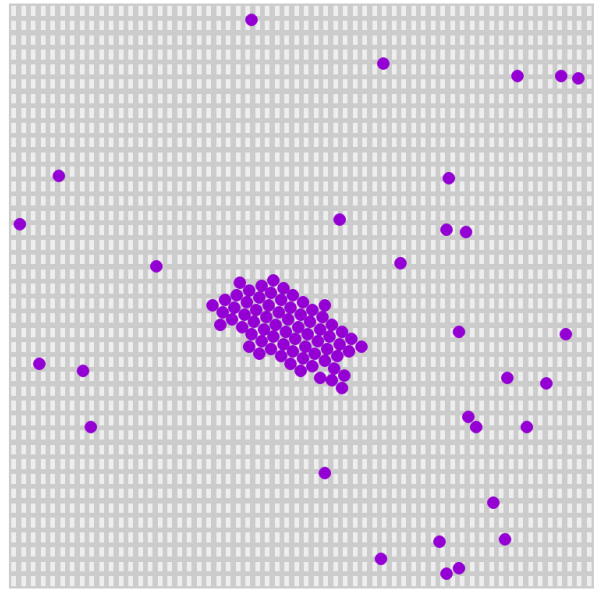

(b)

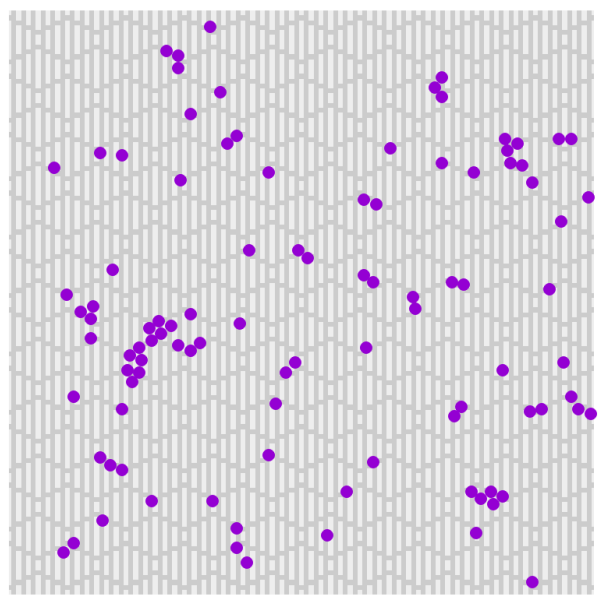

(d)

Fig. 4. Examples of equilibrium spore configurations obtained by Monte Carlo simulations for systems of $N=100$ spores on the smooth (a), Sharklet $n=1$ (b), Sharklet $n=2$ (c) and Sharklet $n=4$ (d) surfaces. The sizes of the surfaces are the same and equal to $240 \times 240 \mu \mathrm{m}^{2}$.

Figure 5(a) shows that the average size of aggregates decreases as $n$ increases from zero but tends to have a constant value for $n \geq 3$. Fig. 5(b) shows that the average number of aggregates increases with $n$ and then saturates for $n \geq 3$. The data for $n=2$ are also close to those for $n=3$. These results indicate that a small number of distinct features, 2 or 3, on the Sharklet pattern are sufficient to provide a strong topographic effect on the aggregation behavior. Increasing the number of distinct features to more than 3 does not give a stronger effect. 

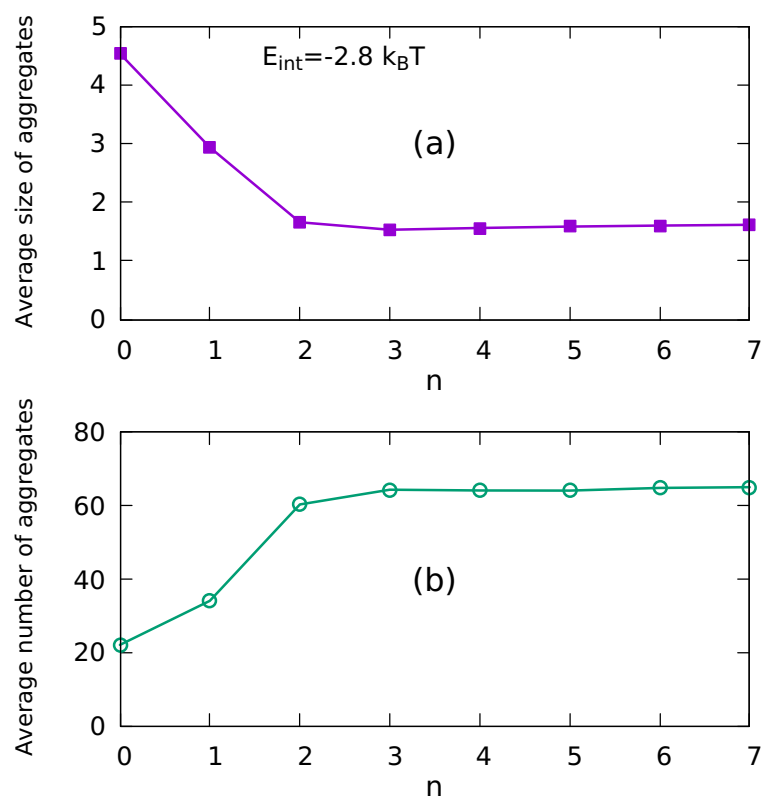

Fig. 5. Dependence of the average size of aggregates (a) and the average number of aggregates (b) on the number of distinct feature $n$ of the Sharklet surfaces obtained for systems of $N=100$ spores with $E_{\text {int }}=-2.8 k_{B} T$. The $n=0$ case corresponds to the smooth surface.

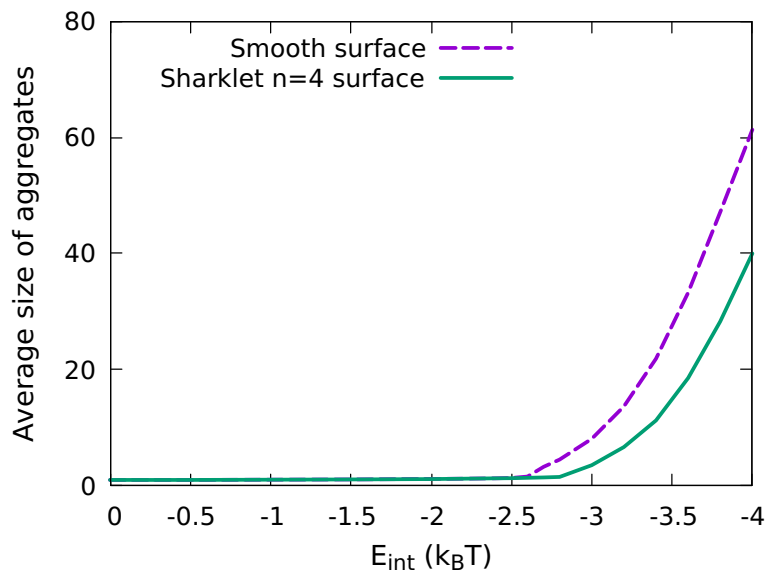

Fig. 6. Dependence of the average size of aggregates on $E_{\text {int }}$ for the systems of $N=100$ spores on smooth (dashed) and Sharklet $n=4$ (solid) surfaces.

In order to check whether the effect of surface topography on aggregation is sensitive to the strength of inter-organism interaction, we calculated the average sizes of aggregates on the surfaces for a range of the energy parameter $E_{\text {int }}$. Fig. 6 shows that for the smooth and the Sharklet 
$n=4$ surfaces the average sizes of the aggregates for the two surfaces are small and almost the same for $E_{\text {int }}>-2.5 k_{B} T$. For $E_{\text {int }}<-2.5 k_{B} T$, the average size of aggregates quickly increases as $E_{\text {int }}$ decreases for both surfaces, but the average size for the smooth surface increases faster and is always larger than that for the Sharklet one. This result indicates that the effect of surface topography on aggregation of microorganisms is always present for a sufficiently large strength of inter-organism interaction. The results shown in Fig. 5 and Fig. 6 are consistent with prior experimental finding that topographic surfaces have a tendency to promote smaller aggregates compared to a smooth surface [21].

\section{CONCLUSION}

Microtopography has an adverse effect on the aggregation of microorganisms fouling on a surface. Using the extended SEA model, this effect can be explained as due to the heterogeneity of the site-dependent attachment propensities induced by the microtopographic pattern. This heterogeneity together with the periodic repetition of the pattern break up large size aggregates to much smaller aggregates when the differences in the attachment energies of an organism for different site types of the surface are sufficiently competitive to the interaction energy between two organisms that make a contact in an aggregate. As a result, the average size of aggregates on a topographic surface is much smaller than on a smooth surface while the number of aggregates is larger. This qualitative effect of surface topography shown by simulations is consistent with experiments. For $U$. linza zoospores on Sharklet surfaces, we have shown that the average size of aggregates decreases when the number of distinct features $n$ increases from zero but quickly saturates when $n \geq 3$. Thus, a small $n$ of at least 3 is sufficient to provide the maximum effect on aggregate sizes. The latter is a quantitative result obtained from the simulations.

Our study shows that the extended SEA model can efficiently predict fouling behaviors of microorganisms on topographic surfaces. One reason for this efficiency is that the energy parameters of the model can be determined by matching the model to an existing experiment allowing quantitative results to be obtained. One can determine the energy parameters using the experiment data for one type of microtopographic pattern. After that, the model can give predictions for any type of microtopographic pattern with the same definitions of site types.

\section{ACKNOWLEDGMENTS}

This research is funded by Vietnam National Foundation for Science and Technology Development (NAFOSTED) under grant number 103.01-2019.363. TXH also thanks for the support of Vietnam Academy of Science and Technology for high-level researchers under task number NVCC05.07/20-20.

\section{REFERENCES}

[1] C. M. Magin, S. P. Cooper and A. B. Brennan, Materials Today 13 (2010) 36.

[2] A. J. Scardino and R. de Nys, Biofouling 27 (2011) 73.

[3] C. M. Kirschner and A. B. Brennan, Annu. Rev. Mater. Res. 42 (2012) 211.

[4] A. Lindholdt, K. Dam-Johansen, S. Olsen, D. M. Yebra and S. Kiil, J. Coat. Technol. Res. 12 (2015) 415.

[5] D. M. Yebra, S. Kiil and K. Dam-Johansen, Prog. Org. Coat. 50 (2004) 75.

[6] K. V. Thomas and S. Brooks, Biofouling 26 (2010) 73. 
[7] M. L. Carman, T. G. Estes, A. W. Feinberg, J. F. Schumacher, W. Wilkerson, L. H. Wilson, M. E. Callow, J. A. Callow and A. B. Brennan, Biofouling 22 (2006) 11.

[8] M. E. Callow, A. R. Jennings, A. Brennan, C. Seegert, A. Gibson, L. Wilson, A. Feinberg, R. Baney and J. Callow, Biofouling 18 (2002) 229.

[9] A. Scardino, E. Harvey and R. De Nys, Biofouling 22 (2006) 55.

[10] A. Scardino, J. Guenther and R. De Nys, Biofouling 24 (2008) 45.

[11] C. J. Long, J. A. Finlay, M. E. Callow, J. A. Callow and A. B. Brennan, Biofouling 26 (2010) 941.

[12] R. N. Wenzel, Ind. Eng. Chem. 28 (1936) 988.

[13] A. Cassie and S. Baxter, Trans. Faraday Soc. 40 (1944) 546.

[14] J. T. Decker, C. M. Kirschner, C. J. Long, J. A. Finlay, M. E. Callow, J. A. Callow and A. B. Brennan, Langmuir 29 (2013) 13023.

[15] T. X. Hoang, H. T. Mai, A. B. Brennan and L. Le, Biofouling 35 (2019) 684.

[16] S. Katharios-Lanwermeyer, C. Xi, N. Jakubovics and A. Rickard, Biofouling 30 (2014) 1235.

[17] H. Dang and C. R. Lovell, Microbiol. Mol. Biol. Rev. 80 (2016) 91.

[18] N. Metropolis, A. W. Rosenbluth, M. N. Rosenbluth, A. H. Teller and E. Teller, J. Chem. Phys. 21 (1953) 1087.

[19] C. J. Long, J. F. Schumacher, P. A. Robinson, J. A. Finlay, M. E. Callow, J. A. Callow and A. B. Brennan, Biofouling 26 (2010) 411.

[20] M. Rovere, D. Heermann and K. Binder, J. Phys. Condens. Matter 2 (1990) 7009.

[21] J. T. Decker, J. T. Sheats and A. B. Brennan, Langmuir 30 (2014) 15212. 